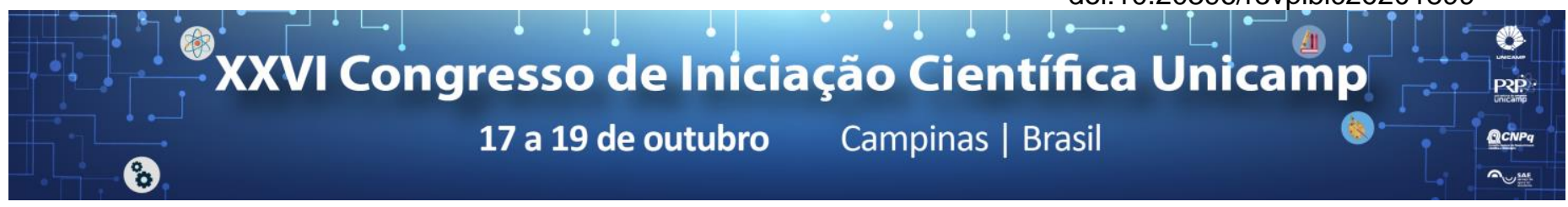

\title{
Hierarchically structured and bifunctional core-shell rare earth nanoparticles
}

\author{
Amanda J. Morais*(IC), Flávia S. Ferreira (PG), Italo O. Mazali (PQ), Fernando A. Sigoli (PQ)
}

\begin{abstract}
Hierarchically nanostructured core@shell systems of NaGdF4:Er:Yb@NaYF4:Yb@NaYF4:Tb:Yb and NaYF4:Tb:Yb@NaYF4:Yb@NaGdF4:Er:Yb composition were synthesized to investigate the influence of composition and crystalline structure on the efficiency of the upconversion emission (UC). The main goal is the preparation of magneto-luminescent systems for a possible application in biological media. Information on the crystalline phase, particle morphologies and the upconversion emission spectra were obtained using: X-Ray Diffraction (XRD), Transmission Electron Microscopy (TEM) and Luminescence Spectroscopy (LS).
\end{abstract}

\section{Key words:}

Nanoparticles, lanthanides, luminescence

\section{Introduction}

Lanthanides ions-doped nanoparticles are currently being studied, mainly because their magnetic and luminescent properties are favorable to obtain useful bifunctional systems, such as magneto-luminescent markers. ${ }^{[1]}$ One of the phenomena responsible for the development of these systems is the possibility of upconversion emission (UC). This phenomenon comprises the absorption of at least two photons of lower energy by the lanthanide ions followed by emission of higher energy photons. ${ }^{[2]}$ To protect the lanthanide ions from non-radiative decay processes, when the nanoparticles are dispersed in a solvent or in a biological environment, a protective shell layer must be deposited over the optical active core leading to the formation of a hierarchically nanostructured core@shell systems.[2][3]This work involves the investigation of the influence of composition and crystalline structure on the efficiency of the emissions in order to obtain a system that exhibits magnetic properties combined with the high efficiency of the UC emissions.

\section{Results and Discussion}

The method used for the synthesis of

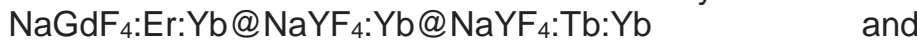
$\mathrm{NaYF}_{4}: \mathrm{Tb}: \mathrm{Yb} @ \mathrm{NaYF}_{4}: \mathrm{Yb} @ \mathrm{NaGdF}_{4}: \mathrm{Er}: \mathrm{Yb}$ was the thermal decomposition of metal precursors in solvents with high boiling points. ${ }^{[4]}$ The trifluoracetates of lanthanides $\left[\mathrm{Ln}\left(\mathrm{OOCCF}_{3}\right)_{3}\right]$ and sodium $\left[\mathrm{NaOOCCF}_{3}\right]$ were used as precursors. The solvents and stabilizers of the synthesis were oleic acid, oleylamine and 1-octadecene. The control of temperature, reaction time and addition of the precursor resulted into the cubic $(\alpha)$ or hexagonal $(\beta)$ crystalline phases. Transmission Electron Microscopy (TEM) images data indicate that the nanoparticles are spheroidal. X-Ray Powder Diffraction (XRD) data show that the control of crystalline phase formation was possible. The upconversion emission spectra show that the crystalline phases influence the emission intensities and electronic transitions. Emission spectra under $980 \mathrm{~nm}$ radiation excitation exhibit the typical emission lines of erbium(III) ion. Finally, the UC emission spectra show that hexagonal crystalline phase has the highest upconversion emission intensity among the prepared nanoparticle systems.

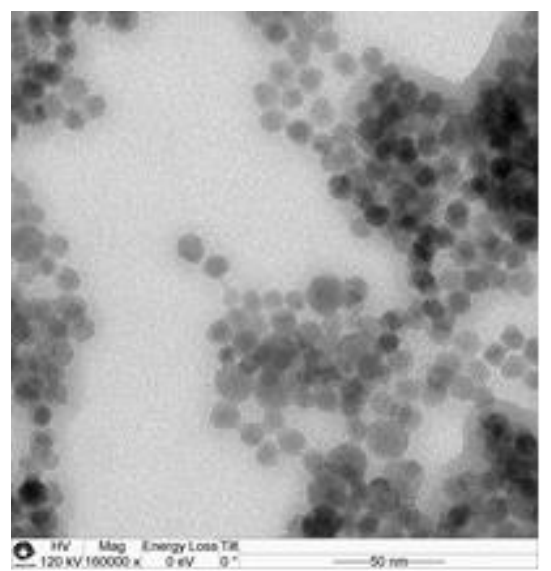

Image 1. Transmission Electron Microscopy image of NaGdF4:Er:Yb@NaYF4:Yb@NaYF4:Tb:Yb nanoparticles.

\section{Conclusions}

The methodology allowed the preparation of spheroidal hierarchically structured rare earth nanoparticles showing higher intensity upconversion emission from hexagonal crystalline phase than cubic ones. The next steps of the research will involve complementary characterizations beyond those already carried out, aiming a better understanding of the systems under investigation. It will also allow a comparative study of their respective photophysical and magnetic properties. A possible nanoparticles surface chemical functionalization will also allow the anchoring of photosensitizer molecules, for singlet oxygen photogeneration to the possible application on photodynamic therapy (PDT).

\section{Acknowledgement}

FAPESP, CNPq, INOMAT and Multi-user Laboratory for Advanced Optical Spectroscopy.

${ }^{1}$ Gautam, A. Veggel; F.C.J.M, Journal of Materials Chemestry, 1, (2013)

${ }^{2}$ Wang, Y. et al, The Journal of Physical Chemistry Letters, 2, (2011)

${ }^{3}$ Johson, N.J.J; Veggel, F.C.J.M. van, Nano Research, 6, 547-561, (2013)

${ }^{4}$ Li, Z.; Zhang, Y., Nanotechnology, 19, (2008) 